\title{
Composite Tracking Control for Generalized Practical Synchronization of Duffing-Holmes Systems with Parameter Mismatching, Unknown External Excitation, Plant Uncertainties, and Uncertain Deadzone Nonlinearities
}

\author{
Yeong-Jeu Sun \\ Department of Electrical Engineering, I-Shou University, Kaohsiung 840, Taiwan \\ Correspondence should be addressed to Yeong-Jeu Sun, yjsun@isu.edu.tw
}

Received 21 February 2012; Accepted 5 April 2012

Academic Editor: Elena Litsyn

Copyright (c) 2012 Yeong-Jeu Sun. This is an open access article distributed under the Creative Commons Attribution License, which permits unrestricted use, distribution, and reproduction in any medium, provided the original work is properly cited.

The generalized practical synchronization (GPS) of uncertain Duffing-Holmes chaotic systems with parameter mismatching, unknown external excitation, plant uncertainties, and uncertain deadzone nonlinearities is investigated. Based on the composite control approach, a tracking control is derived to realize the GPS for the uncertain Duffing-Holmes chaotic systems with parameter mismatching, unknown external excitation, plant uncertainties, and uncertain deadzone nonlinearities. Besides, the guaranteed exponential decay rate, convergence radius, and desired scaling factor can be prespecified. Finally, numerical simulations are provided to illustrate the feasibility and effectiveness of the proposed GPS scheme.

\section{Introduction}

Recently, synchronizations of various dynamic systems or chaotic systems have been intensively studied; see, for instance, [1-6] and the references therein. Occasionally, chaos in many systems is a source of the generation of oscillation. Chaos synchronizations exist in certain fields of application, such as, secure communication, ecological systems, and system identification.

It is well known that there inevitably exist nonlinearities in electric components, such as, operational amplifier, resistor, inductors, capacitor, and electromechanical actuator. Furthermore, the control schemes of controlled driver-response chaotic systems can be realized by various electric components. Input nonlinearities not only often appear in the controlled driver-response chaotic systems but also frequently cause undesirable behavior, 
such as, instabilities or spurious limit cycles. Over the past decades, researchers have been concerned with several input nonlinearities common in dynamical systems, such as, deadzones, saturation, hysteresis, relays, and others; see, for instance, [7-10]. Generally speaking, the synchronization of chaotic system with parameter mismatching, unknown external excitation, plant uncertainties, and uncertain deadzone nonlinearities is in general not as simple as that without any uncertainties and nonlinearities.

Over the past decades, Duffing systems have been received a great deal of interest due to theoretical interests and successful applications in numerous areas. In [11], the virtual stabilizability of Duffing-Holmes control systems has been studied and a tracking control has been proposed such that the states of Duffing-Holmes control system track the desired trajectories. In addition, a harmonic balance method in conjunction with the successive integration technique has been offered in [12] to solve the Duffing oscillator equation with damping and excitation. In this paper, the concept of the GPS for chaotic systems is presented and the GPS of uncertain Duffing-Holmes chaotic systems with parameter mismatching, unknown external excitation, plant uncertainties, and uncertain deadzone nonlinearities is explored. Using the composite control strategy, a tracking control is offered to realize the GPS for uncertain Duffing-Holmes chaotic systems, with any prespecified exponential decay rate, convergence radius, and desired scaling factor.

\section{Problem Formulation and Main Result}

Before presenting the problem formulation, let us introduce a definition as follows.

Definition 2.1. The deadzone nonlinearities $D(u, \bar{d}, m)$, with $\bar{d} \geq 0$ and $m>0$, is defined to be the collection of all functions $\Delta \phi(u):=\mathfrak{R} \rightarrow \mathfrak{R}$ satisfying

$$
\Delta \phi(u):= \begin{cases}m(u-\Delta \bar{r}), & \text { if } u \geq \Delta \bar{r} \\ 0, & \text { if }-\Delta \underline{r} \leq u \leq \Delta \bar{r} \\ m(u+\Delta \underline{r}) & \text { if } u \leq-\Delta \underline{r}\end{cases}
$$

for any $\Delta \bar{r}$ and $\Delta \underline{r}$ with $0 \leq \Delta \underline{r}, \Delta \bar{r} \leq \bar{d}$.

In this paper, we consider the following uncertain Duffing-Holmes chaotic systems with parameter mismatching, unknown external excitation, plant uncertainties, and uncertain deadzone nonlinearities described as

Driver system:

$$
\begin{gathered}
\ddot{r}+p_{1} r+p_{2} \dot{r}+r^{3}+p_{3} \cos (w t)+\Delta f_{1}(t, r, \dot{r})=0, \quad t \geq 0, \\
{\left[\begin{array}{cc}
r(0) & \dot{r}(0)
\end{array}\right]=\left[\begin{array}{ll}
r_{i 1} & r_{i 2}
\end{array}\right],}
\end{gathered}
$$

Response system:

$$
\begin{aligned}
& \ddot{q}+p_{1} q+p_{2} \dot{q}+\Delta f_{2}(t, q, \dot{q})+\Delta \phi(u)=0, \quad t \geq 0,
\end{aligned}
$$

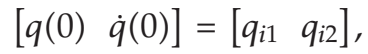


where $r, q \in \mathfrak{R}, \Delta f_{1}$ and $\Delta f_{2}$, for all $i \in\{1,2\}$, represent the mixed uncertainties (parameter mismatching, unknown external excitation, and plant uncertainties), $\left[\begin{array}{lll}r_{i 1} & r_{i 2}\end{array}\right]$ and $\left[\begin{array}{ll}q_{i 1} & q_{i 2}\end{array}\right]$ represent the initial conditions, $u \in \mathfrak{R}$ is the control input, and $\Delta \phi(u) \in D(u, \bar{d}, m)$ is the uncertain deadzone nonlinearity. For the existence of the solutions of (2.2), we assume that the uncertainties $\Delta f_{1}$ and $\Delta f_{2}$ are continuous. It is noted that the system (2.2) without any uncertainties (i.e., $\Delta f_{1}=\Delta f_{2}=0$ ) displays chaotic behavior for certain values of the parameters [13]. Letting

$$
\begin{aligned}
& x(t):=\left[\begin{array}{ll}
x_{1}(t) & x_{2}(t)
\end{array}\right]^{T}:=\left[\begin{array}{ll}
r(t) & \dot{r}(t)
\end{array}\right]^{T}, \\
& z(t):=\left[\begin{array}{ll}
z_{1}(t) & z_{2}(t)
\end{array}\right]^{T}:=\left[\begin{array}{ll}
q(t) & \dot{q}(t)
\end{array}\right]^{T},
\end{aligned}
$$

the corresponding state-space equations of system (2.2) and system (2.3) are

$$
\begin{gathered}
\dot{x}_{1}=x_{2}, \\
\dot{x}_{2}=-p_{1} x_{1}-p_{2} x_{2}-x_{1}^{3}-p_{3} \cos (w t)-\Delta f_{1}\left(t, x_{1}, x_{2}\right), \quad \forall t \geq 0, \\
\dot{z}_{1}=z_{2}, \\
\dot{z}_{2}=-p_{1} z_{1}-p_{2} z_{2}-\Delta f_{2}\left(t, z_{1}, z_{2}\right)-\Delta \phi(u), \quad \forall t \geq 0 .
\end{gathered}
$$

Throughout this paper, an assumption is made as follows.

(A1) There exist two continuous functions $g_{1}(t, r, \dot{r}) \geq 0$ and $g_{2}(t, q, \dot{q}) \geq 0$ such that, for all arguments,

$$
\left|\Delta f_{1}(t, r, \dot{r})\right| \leq g_{1}(t, r, \dot{r}), \quad\left|\Delta f_{2}(t, q, \dot{q})\right| \leq g_{2}(t, q, \dot{q})
$$

For brevity, let us define the synchronous error vector as

$$
e(t):=\left[\begin{array}{ll}
e_{1}(t) & e_{2}(t)
\end{array}\right]^{T}:=z(t)-\beta x(t),
$$

where $\beta \in \mathfrak{R}$ is the scaling factor.

The purpose of this paper is to search a composite control $u=u_{1}+u_{2}$ such that the states $z_{1}$ and $z_{2}$ of the response system (2.6) track, respectively, the signals $\beta x_{1}$ and $\beta x_{2}$ of the driver system (2.5), with any desired scaling factor $\beta$.

The precise definition of the GPS, in terms of error response, is given as follows.

Definition 2.2. The driver-response dynamical systems (2.5) and (2.6) are said to realize the GPS, provided that, for any $\alpha>0, \varepsilon>0$, and $\beta \in \mathfrak{R}$, there exists a tracking control $u(t):=$ $u(\alpha, \varepsilon, \beta)$ and positive number $k$ such that the synchronous error satisfies

$$
\|e(t)\| \leq k \cdot e^{-\alpha t}+\varepsilon, \quad \forall t \geq 0 .
$$

In this case, the positive number $\varepsilon$ is called the convergence radius, the positive number $\alpha$ is called the exponential decay rate, and $\beta$ is called the desired scaling factor. In other words, the 
GPS means that the states $z_{1}$ and $z_{2}$ of the response system can track, respectively, the signals $\beta x_{1}$ and $\beta x_{2}$ of the driver system, with any prespecified exponential decay rate, convergence radius, and desired scaling factor.

From (2.5)-(2.8), we have the following error dynamical system:

$$
\begin{aligned}
\dot{e} & =\left[\begin{array}{cc}
0 & 1 \\
-p_{1} & -p_{2}
\end{array}\right] e+B\left[\begin{array}{c}
0 \\
-\Delta \phi-\Delta f_{2}+\beta x_{1}^{3}+\beta p_{3} \cos (w t)+\beta \Delta f_{1}
\end{array}\right] \\
& =\left[\begin{array}{cc}
0 & 1 \\
-p_{1} & -p_{2}
\end{array}\right] e-m B u+B\left[\begin{array}{c}
0 \\
\Delta f
\end{array}\right], \quad \forall t \geq 0
\end{aligned}
$$

with $B:=\left[\begin{array}{l}0 \\ 1\end{array}\right]$ and $\Delta f:=[-\Delta \phi(u)+m u]+\beta x_{1}^{3}+\beta p_{3} \cos (w t)+\beta \Delta f_{1}-\Delta f_{2}$. First consider the case of the system (2.10) without any parameter mismatching, unknown external excitation, and plant uncertainties, that is, $\Delta f=0$. Thus the nominal system of (2.10) can represented as

$$
\dot{e}=\left[\begin{array}{cc}
0 & 1 \\
-p_{1} & -p_{2}
\end{array}\right] e-m B u
$$

According to the linear system theory, it can be easily obtained that given any positive constant $\alpha$, the system (2.11) subjected to the linear control law:

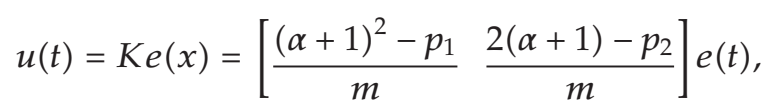

is globally exponentially stable with guaranteed exponential decay rate $\alpha$. Nevertheless, the nominal control law (2.12) may not suffice to render the uncertain error system (2.10) to be globally exponentially stable. Hence a corrective control term must be added to overcome the uncertain part of (2.10). In the following, a composite control, consisting of a nominal control and a corrective control, is proposed such that the GPS between systems (2.5) and (2.6) can be guaranteed.

Now we present the main result for the GPS between system (2.5) and system (2.6).

Theorem 2.3. The GPS between system (2.5) and system (2.6) can be achieved under the composite control $u(t):=u(\alpha, \varepsilon, \beta)$ defined by

$$
u(t)=u_{1}(t)+u_{2}(t)
$$

where

$$
\begin{gathered}
u_{1}(t)=K e(x)=\left[\frac{(\alpha+1)^{2}-p_{1}}{m} \frac{2(\alpha+1)-p_{2}}{m}\right] e(t), \\
u_{2}(t)=\frac{h^{2}(t)}{m \cdot h(t) \cdot\left|p_{21} e_{1}+p_{22} e_{2}\right|+\alpha \cdot m \cdot \varepsilon^{2} \cdot \lambda_{\min }(P)}\left(p_{21} e_{1}+p_{22} e_{2}\right),
\end{gathered}
$$


where

$$
h(t)=m \bar{d}+|\beta| \cdot g_{1}+g_{2}+\left|\beta x_{1}^{3}\right|+\left|\beta p_{3}\right|
$$

and $P:=\left\lfloor p_{i j}\right\rfloor_{2 \times 2}>0$ is the unique solution to the following Lyapunov equation:

$$
(A+\alpha I)^{T} P+P(A+\alpha I)=-2 I,
$$

with $A:=\left[\begin{array}{cc}0 & 1 \\ -(\alpha+1)^{2} & -2(\alpha+1)\end{array}\right]$.

Proof. From (2.10), (2.13), and (2.14), the dynamical error system can be performed

$$
\begin{aligned}
\dot{e} & =\left[\begin{array}{cc}
0 & 1 \\
-p_{1} & -p_{2}
\end{array}\right] e-m B\left(u_{1}+u_{2}\right)+B\left[\begin{array}{c}
0 \\
\Delta f
\end{array}\right] \\
& =A e-m B u_{2}+B\left[\begin{array}{c}
0 \\
\Delta f
\end{array}\right], \quad \forall t \geq 0,
\end{aligned}
$$

with $B:=\left[\begin{array}{l}0 \\ 1\end{array}\right]$ and $\Delta f:=[m u-\Delta \phi(u)]+\beta x_{1}^{3}+\beta p_{3} \cos (w t)+\beta \Delta f_{1}-\Delta f_{2}$.

Obviously, one has $\sigma(A)=\{-\alpha-1\}, \sigma(A+\alpha I)=\{-1\}$, and $|\Delta f| \leq h(t)$. Let

$$
W(e(t))=e^{T}(t) P e(t) .
$$

The time derivative of $W(e(t))$ along the trajectories of dynamical error system, with (2.11)(2.19) and (A1), is given by

$$
\begin{aligned}
\dot{W}(e(t)) & =\dot{e}^{T} P e+e^{T} P \dot{e} \\
& =\left[A e+B\left(-m u_{2}+\Delta f\right)\right]^{T} P e+e^{T} P\left[A e+B\left(-m u_{2}+\Delta f\right)\right] \\
& =e^{T}\left(A^{T} P+P A\right) e-2 m \cdot e^{T} P B u_{2}+2 e^{T} P B \cdot \Delta f \\
& =e^{T}(-2 \alpha P-2 I) e-2 m \cdot e^{T} P B u_{2}+2 e^{T} P B \cdot \Delta f \\
& \leq-2 \alpha W-2 m \cdot e^{T} P B u_{2}+2\left|e^{T} P B\right| \cdot|\Delta f| \\
& \leq-2 \alpha W-2 \frac{h^{2}(t)}{h \cdot\left|B^{T} P e(t)\right|+\alpha \cdot \varepsilon^{2} \cdot \lambda_{\min }(P)}\left|e^{T} P B\right|^{2}+2\left|e^{T} P B\right| \cdot h \\
& =-2 \alpha W+2 \frac{\alpha \cdot \varepsilon^{2} \cdot \lambda_{\min }(P) \cdot h \cdot\left|e^{T} P B\right|}{h \cdot\left|B^{T} P e(t)\right|+\alpha \cdot \varepsilon^{2} \cdot \lambda_{\min }(P)} \\
& =-2 \alpha W+2 \alpha \cdot \varepsilon^{2} \cdot \lambda_{\min }(P), \quad \forall t \geq 0,
\end{aligned}
$$

in view of $|\Delta f(t)| \leq h(t)$ and $p_{21} e_{1}+p_{22} e_{2}=B^{T} P e=e^{T} P B$. Thus, one has

$$
e^{2 \alpha_{1} t} \cdot \dot{W}+e^{2 \alpha t} \cdot 2 \alpha W=\frac{d}{d t}\left[e^{2 \alpha t} \cdot W\right] \leq e^{2 \alpha t} \cdot 2 \alpha \cdot \varepsilon^{2} \cdot \lambda_{\min }(P), \quad \forall t \geq 0
$$


It follows that

$$
\begin{aligned}
\int_{0}^{t} \frac{d}{d t}\left[e^{2 \alpha t} \cdot W(e(t))\right] d t & =e^{2 \alpha t} \cdot W(e(t))-W(e(0)) \\
& \leq \int_{0}^{t} e^{2 \alpha t} \cdot 2 \alpha \cdot \varepsilon^{2} \cdot \lambda_{\min }(P) d t \\
& =\varepsilon^{2} \cdot \lambda_{\min }(P)\left(e^{2 \alpha t}-1\right), \quad \forall t \geq 0 .
\end{aligned}
$$

This implies

$$
W(e(t)) \leq\left[W(e(0))-\varepsilon^{2} \cdot \lambda_{\min }(P)\right] \cdot e^{-2 \alpha t}+\varepsilon^{2} \cdot \lambda_{\min }(P), \quad \forall t \geq 0
$$

Thus, from (2.19) and (2.23), it can be readily obtained that

$$
\lambda_{\min }(P)\|e(t)\|^{2} \leq W(e(t)) \leq e^{-2 \alpha t}\left[W(e(0))-\varepsilon^{2} \lambda_{\min }(P)\right]+\varepsilon^{2} \lambda_{\min }(P), \quad \forall t \geq 0 .
$$

Consequently, we conclude that

$$
\begin{aligned}
\|e(t)\| & \leq \sqrt{e^{-2 \alpha t}\left[\frac{W(e(0))+\varepsilon^{2} \lambda_{\min }(P)}{\lambda_{\min }(P)}\right]}+\varepsilon^{2} \\
& \leq \sqrt{e^{-2 \alpha t\left[\frac{W(e(0))+\varepsilon^{2} \lambda_{\min }(P)}{\lambda_{\min }(P)}\right]}+\sqrt{\varepsilon^{2}}} \\
& =\sqrt{\left[\frac{W(e(0))+\varepsilon^{2} \lambda_{\min }(P)}{\lambda_{\min }(P)}\right]} \cdot e^{-\alpha t}+\varepsilon, \quad \forall t \geq 0 .
\end{aligned}
$$

This completes the proof.

Remark 2.4. In what follows, we present an algorithm to find a tracking control law of (2.13) stated in Theorem 2.3.

INPUT: the driver-response systems (2.5) and (2.6), the prespecified exponential decay rate $\alpha>0$, and the prespecified convergence radius $\varepsilon>0$.

OUPUT: tracking control of (2.13).

Step 1. Choose $g_{1}(t, r, \dot{r})$ and $g_{2}(t, q, \dot{q})$ such that (A1) is satisfied.

Step 2. Calculate $m$ and $\bar{d}$, from Definition 2.1.

Step 3. Calculate $P, \lambda_{\min }(P), p_{21}$, and $p_{22}$, from (2.17).

Step 4. Form $e_{1}$ and $e_{2}$ from (2.8). 


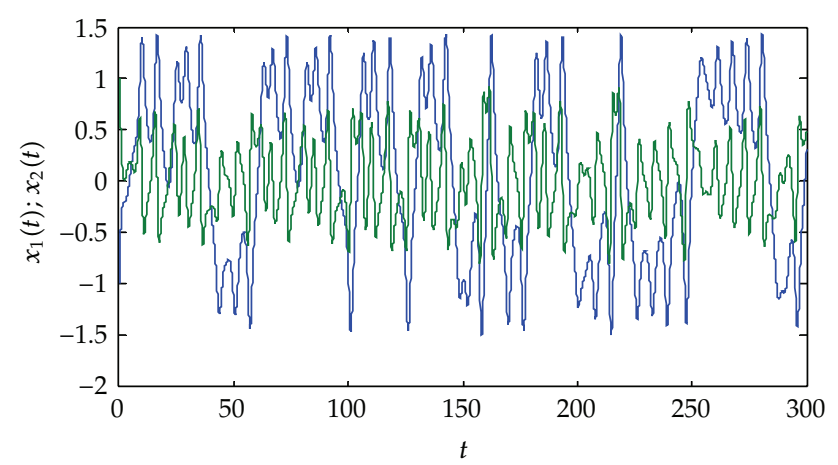

Figure 1: Typical state trajectories of the uncertain Duffing-Holmes system for (3.1).

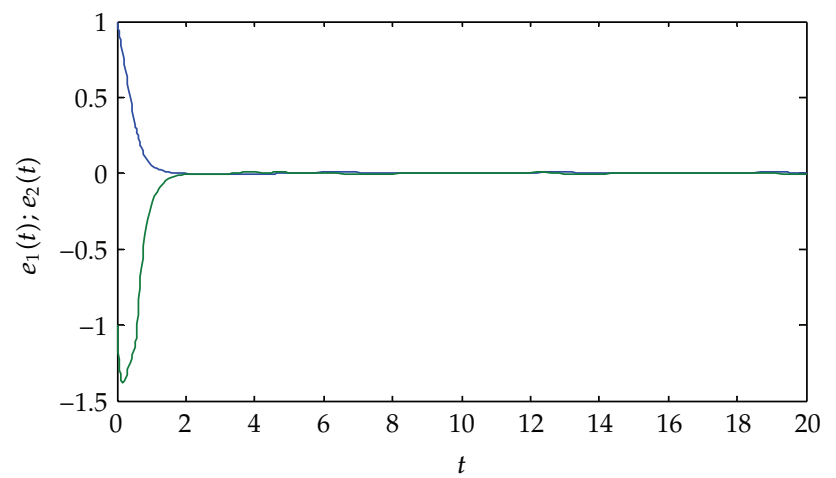

Figure 2: Synchronization errors of systems (3.1)-(3.7) with $\beta=1$.

Step 5. Form $u_{1}$ from (2.14).

Step 6. Form $h(t)$ from (2.16).

Step 7. Form $u_{2}$ from (2.15).

Step 8. OUPUT $u(t)=u_{1}(t)+u_{2}(t)$.

\section{Illustrative Example}

In what follows, we provide an example to illustrate the main result.

Driver system:

$$
\begin{gathered}
\dot{x}_{1}=x_{2}, \\
\dot{x}_{2}=-p_{1} x_{1}-p_{2} x_{2}-x_{1}^{3}-p_{3} \cos (w t)-\Delta a(t) x_{1} x_{2}, \quad \forall t \geq 0 .
\end{gathered}
$$




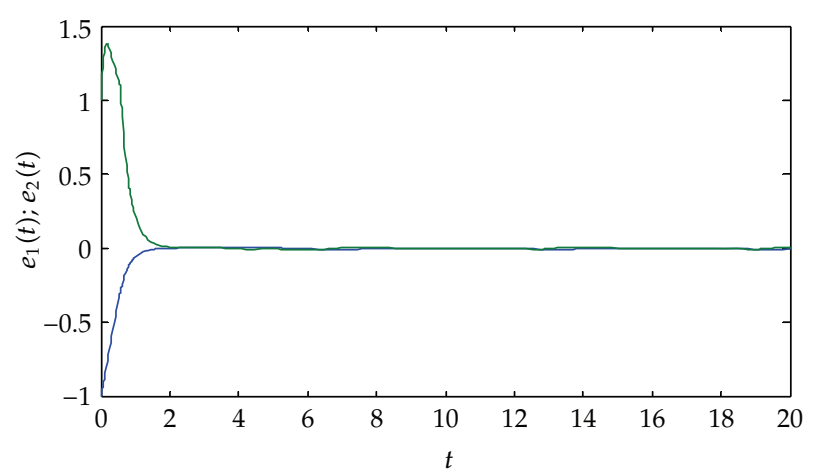

Figure 3: Synchronization errors of systems (3.1)-(3.7) with $\beta=-1$.

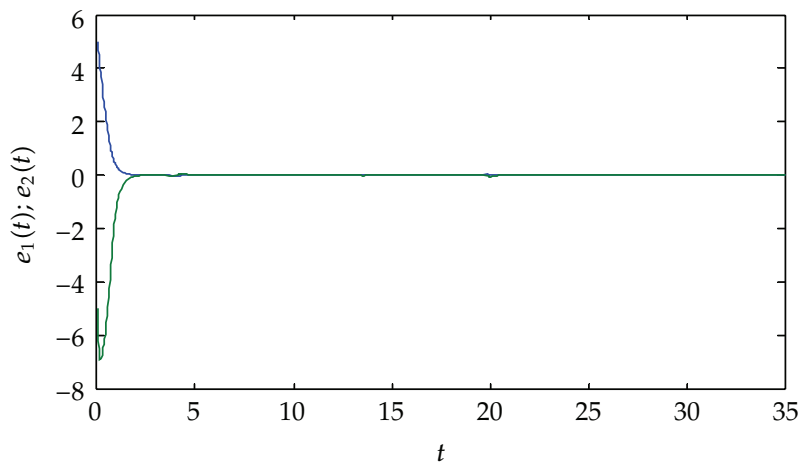

Figure 4: Synchronization errors of systems (3.1)-(3.7) with $\beta=5$.

Response system:

$$
\begin{gathered}
\dot{z}_{1}=z_{2} \\
\dot{z}_{2}=-p_{1} z_{1}-p_{2} z_{2}-\Delta b(t) \sin (t)-\Delta \phi(u), \quad \forall t \geq 0,
\end{gathered}
$$

where

$$
\begin{aligned}
p_{1}=-1, & p_{2}=0.25 \quad p_{3}=0.3, \quad w=1, \\
-0.1 \leq \Delta a(t) \leq 0.1, & -0.1 \leq \Delta b(t) \leq 0.1, \quad \Delta \phi(u) \in D(u, 1,2) .
\end{aligned}
$$

Our objective, in this example, is to design a tracking control such that the systems (3.1) and (3.2) can realize the GPS, with the exponential decay rate $\alpha=2$ and convergence radius $\varepsilon=0.1$. Comparison of (3.1)-(3.2) with (2.5)-(2.6), it can be obtained that

$$
\begin{aligned}
\bar{d}=1, & m=2, \\
\Delta f_{1}\left(t, x_{1}, x_{2}\right)=\Delta a(t) \cdot x_{1} x_{2}, \quad & \Delta f_{2}\left(t, z_{1}, z_{2}\right)=\Delta b(t) \sin (t) .
\end{aligned}
$$




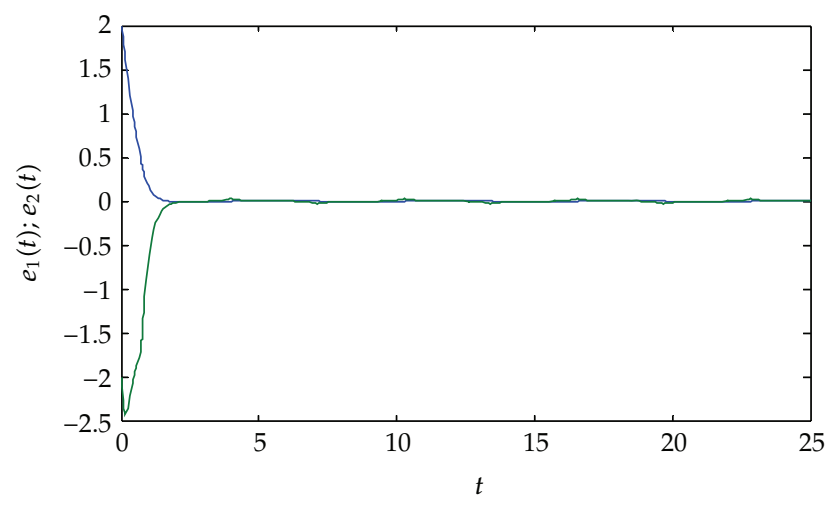

Figure 5: Synchronization errors of systems (3.1)-(3.7) with $\beta=0$.

The condition (A1) is evidently satisfied if we let

$$
g_{1}\left(t, x_{1}, x_{2}\right)=0.1 \cdot\left|x_{1} x_{2}\right|, \quad g_{2}\left(t, z_{1}, z_{2}\right)=0.1 \cdot|\sin (t)| .
$$

Solving the Lyapunov equation (2.17) yields

$$
\begin{array}{cl}
P=\left[\begin{array}{cc}
49 & 11 \\
11 & 3
\end{array}\right], \quad & \lambda_{\min }(P)=0.5049, \\
p_{21}=11, & p_{22}=3 .
\end{array}
$$

Finally, owing to (2.13)-(2.16), we obtain the design controller

$$
u(t)=5 e_{1}+2.875 e_{2}+\frac{h^{2}(t)}{2 \cdot h(t) \cdot\left|11 e_{1}+3 e_{2}\right|+0.02}\left(11 e_{1}+3 e_{2}\right),
$$

with

$$
h(t)=2+0.1 \cdot\left[|\beta| \cdot\left|x_{1} x_{2}\right|+|\sin (t)|\right]+\left|\beta x_{1}^{3}\right|+|0.3 \beta| .
$$

Consequently, by Theorem 2.3, we conclude that the GPS between the driver system (3.1) and the response system (3.2) can be achieved under the control law (3.7), with the guaranteed exponential decay rate $\alpha=2$ and convergence radius $\varepsilon=0.1$.

With, for instance,

$$
\Delta a(t)=\Delta b(t)=0.1
$$

the typical state trajectories of uncertain Duffing-Holmes chaotic system (3.1) are depicted in Figure 1. In addition, the synchronization errors of systems (3.1)-(3.7) with $\beta=1,-1,5$, and 0 , are depicted in Figures 2, 3, 4 and 5, respectively. From the foregoing simulations results, it is seen that the controlled drive-response systems (3.1) and (3.2) achieve the GPS under the control law (3.7). 


\section{Conclusion}

In this paper, the GPS of uncertain Duffing-Holmes chaotic systems with parameter mismatching, unknown external excitation, plant uncertainties, and uncertain deadzone nonlinearities has been investigated. Based on the composite control methodology, a tracking control has been proposed to realize the GPS for such uncertain Duffing-Holmes chaotic systems, with prespecified exponential decay rate, convergence radius, and desired scaling factor. Finally, numerical simulations have also been given to verify the feasibility and effectiveness of the proposed GPS scheme.

\section{Nomenclature}

$\Re^{n}$ : $\quad$ The $n$-dimensional real space

$|a|$ : $\quad$ The modulus of a real number $a$

$\|x\|: \quad$ The Euclidean norm of the vector $x \in \mathfrak{R}^{n}$

I: $\quad$ The unit matrix

$A^{T}: \quad$ The transport of the matrix $A$

$\lambda_{\text {min }}(P)$ : The minimum eigenvalue of the matrix

$P$ with real eigenvalues

$\sigma(A): \quad$ The spectrum of the matrix $A$

$P>0$ : The matrix $P$ is a symmetric positive definite matrix.

\section{Acknowledgments}

The author thanks the National Science Council of Republic of China for supporting this work under Grant NSC-100-2221-E-214-015. The author also wishes to thank the I-Shou University for supporting this work under Grant ISU100-01-01.

\section{References}

[1] X.-L. An, J.-N. Yu, Y.-Z. Li, Y.-D. Chu, J.-G. Zhang, and X.-F. Li, “Design of a new multistage chaos synchronized system for secure communications and study on noise perturbation," Mathematical and Computer Modelling, vol. 54, no. 1-2, pp. 7-18, 2011.

[2] J. Banasiak, "Chaos in Kolmogorov systems with proliferation-general criteria and applications," Journal of Mathematical Analysis and Applications, vol. 378, no. 1, pp. 89-97, 2011.

[3] Y.-Q. Che, J. Wang, S.-G. Cui et al., "Chaos synchronization of coupled neurons via adaptive sliding mode control," Nonlinear Analysis, vol. 12, no. 6, pp. 3199-3206, 2011.

[4] S. Gakkhar and A. Singh, "Control of chaos due to additional predator in the Hastings-Powell food chain model," Journal of Mathematical Analysis and Applications, vol. 385, no. 1, pp. 423-438, 2012.

[5] N. Jia and T. Wang, "Chaos control and hybrid projective synchronization for a class of new chaotic systems," Computers \& Mathematics with Applications, vol. 62, no. 12, pp. 4783-4795, 2011.

[6] Y. Yang, Y. Wang, X. Yuan, and F. Yin, "Hybrid chaos optimization algorithm with artificial emotion," Applied Mathematics and Computation, vol. 218, no. 11, pp. 6585-6611, 2012.

[7] D. H. Ji, J. H. Park, and S. C. Won, "Master-slave synchronization of Lur'e systems with sector and slope restricted nonlinearities," Physics Letters A, vol. 373, no. 11, pp. 1044-1050, 2009.

[8] H. Kebriaei and M. Javad Yazdanpanah, "Robust adaptive synchronization of different uncertain chaotic systems subject to input nonlinearity," Communications in Nonlinear Science and Numerical Simulation, vol. 15, no. 2, pp. 430-441, 2010. 
[9] J. Li, W. Li, and Q. Li, "Sliding mode control for uncertain chaotic systems with input nonlinearity," Communications in Nonlinear Science and Numerical Simulation, vol. 17, no. 1, pp. 341-348, 2012.

[10] X. Wu, X. Wu, X. Luo, Q. Zhu, and X. Guan, "Neural network-based adaptive tracking control for nonlinearly parameterized systems with unknown input nonlinearities," Neurocomputing, vol. 82, no. 1, pp. 127-142, 2012.

[11] Y. J. Sun, "Robust tracking control of uncertain Duffing-Holmes control systems," Chaos, Solitons $\mathcal{E}$ Fractals, vol. 40, no. 3, pp. 1282-1287, 2009.

[12] Y. Z. Chen and X. Y. Lin, "A successive integration technique for the solution of the Duffing oscillator equation with damping and excitation," Nonlinear Analysis A, vol. 70, no. 10, pp. 3603-3608, 2009.

[13] S. Bowong, F. M. Moukam Kakmeni, and J. L. Dimi, "Chaos control in the uncertain Duffing oscillator," Journal of Sound and Vibration, vol. 292, no. 3-5, pp. 869-880, 2006. 


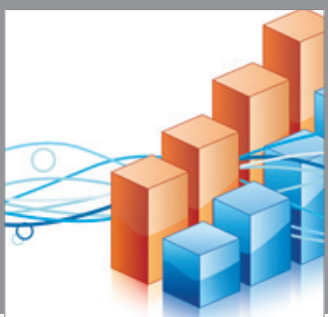

Advances in

Operations Research

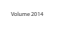

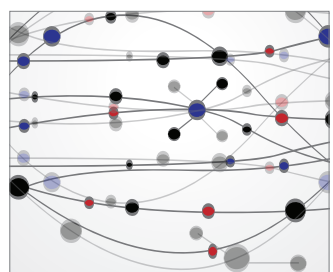

\section{The Scientific} World Journal
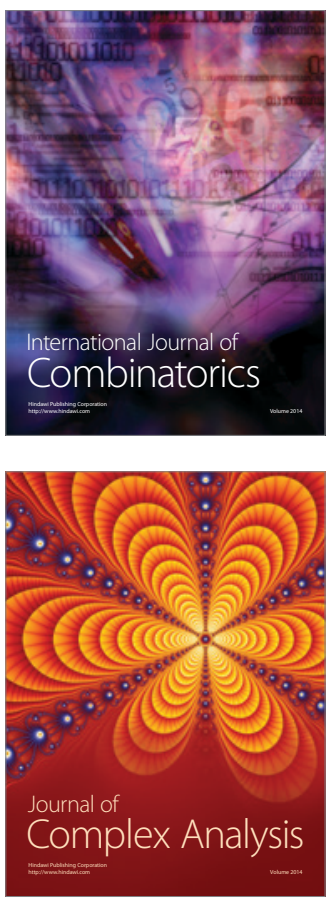

International Journal of

Mathematics and

Mathematical

Sciences
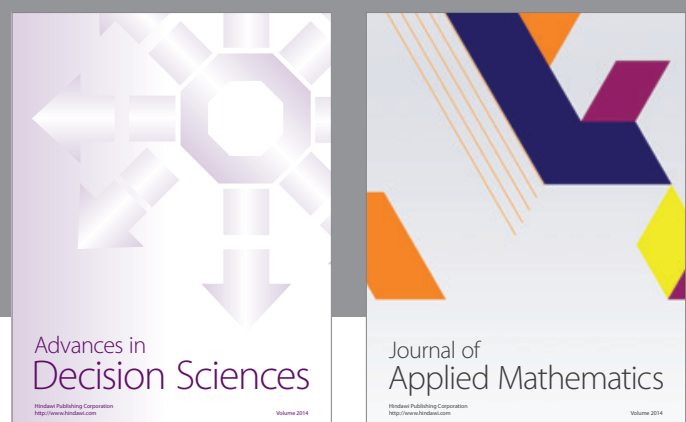

Journal of

Applied Mathematics
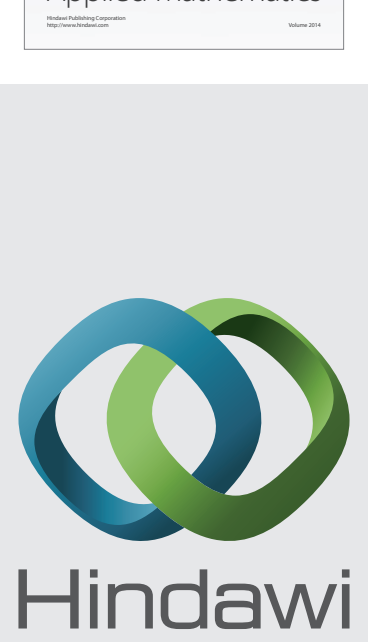

Submit your manuscripts at http://www.hindawi.com
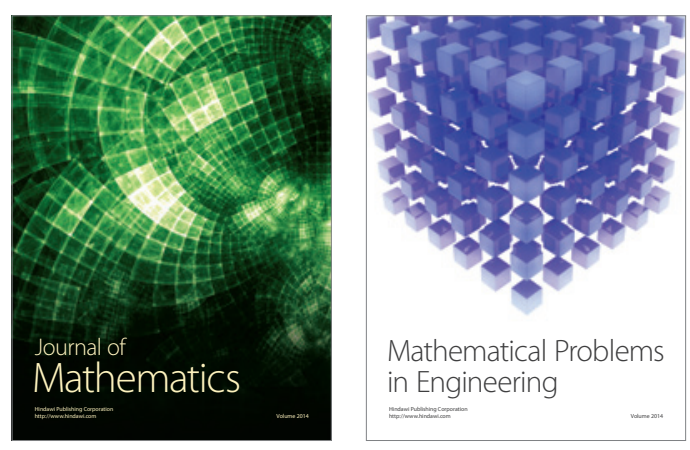

Mathematical Problems in Engineering
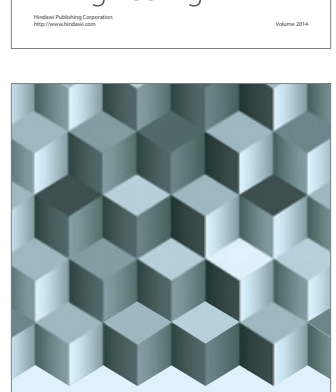

Journal of

Function Spaces
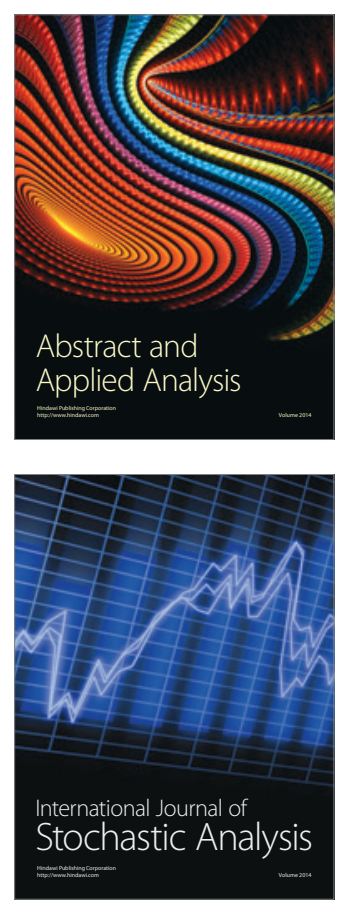

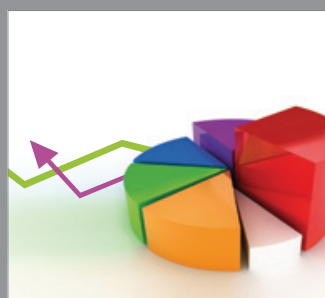

ournal of

Probability and Statistics

Promensencen
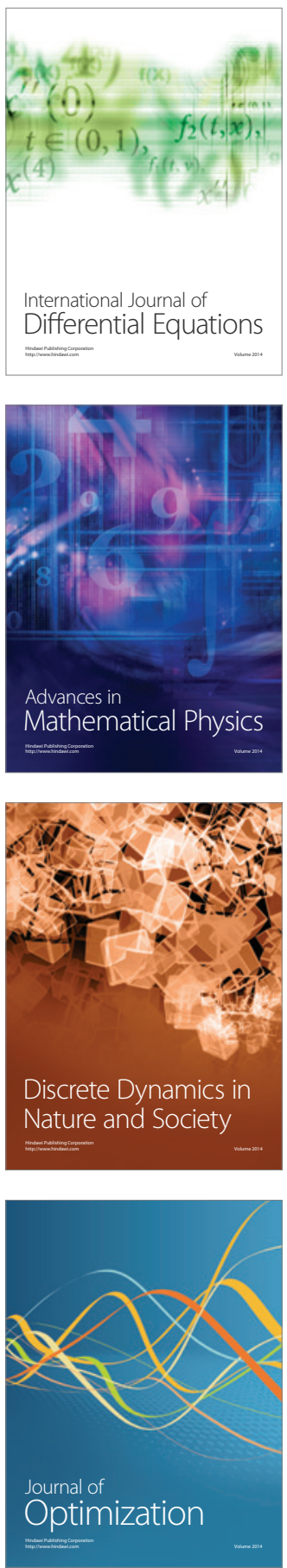